\title{
Notes on Inequalities.
}

By V. Ramaswami, M.A.

[The following is a digest, consisting mainly of extracts, of $\mathrm{Mr}$ Ramaswami's paper. The author mentions that the Notes are intended for readers of Chrystal's " Algebra."]

On a General Inequality Theorem.

1. The important inequality of which Prof. Chrystal has given so many examples may be called the "Power Inequality."

There is a simple theorem of the Differential Calculus which is to a general function $f(x)$, what the Power Inequality is to the function $x^{m}$.

In what follows it will be supposed that the functions and the differential coefficients considered are finite, single-valued, and continuous between the limits of the variable considered, though they may be infinite, at either limit.

2. Theorem: If $f^{\prime \prime}(x)$ be always positive, or always negative, as $x$ increases from a value $B$ to $a$ value $A$, and if $a$ and $b$ be any two quantities lying between the limits $\mathrm{A}$ and $\mathrm{B}, a$ being greater than $b$, then

$$
f^{\prime}(a) \geqslant \frac{f(a)-f(b)}{a-b} \geqslant f^{\prime}(b)
$$

according as $f^{\prime \prime}(x) \geqslant 0$, between the limits $A$ and $B$.

[Then follow several proofs of the theorem (which is practically an aspect of the Mean Value Theorem); the simplest is that obtained from consideration of the fact that the curve $y=f(x)$ is, under the specitied conditions, either convex or concave to the axis of $x$ throughout the range of values considered.]

Applying the theorem to the elementary functions, we have

(i) If $x$ and $y$ be positive, and $x>y$, then

$$
m x^{m-1} \geqslant \frac{x^{n}-y^{m}}{x-y} \geqslant m y^{m-1}
$$

according as $m(m-1) \geqslant 0$. (The Power-Inequality.) 
(ii) If $a$ is any positive quantity $\neq 1$, and $x>y$,

$$
a^{x} \log a>\frac{a^{x}-a^{y}}{x-y}>a^{y} \log a .
$$

(iii) If $x$ and $y$ be positive, and $x>y$,

(iv) If $\frac{\pi}{2}>x>y>0$,

$$
\frac{1}{x}<\frac{\log x-\log y}{x-y}<\frac{1}{y} .
$$

$$
\begin{gathered}
\cos x<\frac{\sin x-\sin y}{x-y}<\cos y ; \\
\text { etc. }
\end{gathered}
$$

We proceed to deduce some consequences from the general theorem.

3. Theorem: If $f^{\prime \prime}(x)$ be constantly positive, or constantly negative, as $x$ increases from $\mathrm{B}$ to $\mathrm{A}$, and if $x, y, z$ be sny three quantities in descending order of magnitude, lying between the limits $A$ and $B$, then

$$
f(x) \cdot(y-z)+f(y) \cdot(z-x) \cdot)+f(z) \cdot(x-y) \geqslant 0,
$$

according as $f^{\prime \prime}(x) \geqslant 0$, between the limits $A$ and $B$.

Demonstration: Suppose $f^{\prime \prime}(x)$ to be positive. Then, by the general theorem,

$$
\begin{aligned}
& \quad \frac{f(x)-f(y)}{x-y}>f^{\prime}(y)>\frac{f(y)-f(z)}{y-z} ; \\
& \therefore \frac{f(x)-f(y)}{x-y}>\frac{f(y)-f(z)}{y-z} .
\end{aligned}
$$

The denominators being positive, we have multiplying out, etc., the result

$$
f(x) \cdot(y-z)+f(y) \cdot(z-x)+f(z) \cdot(x-y)>0 .
$$

If $f^{\prime \prime}(x)$ be negative, the inequality sign is reversed throughout.

$$
\text { Examples: (i) } f(x)=a^{x}, \quad \text { (ii) } f(x)=x^{m}, \quad \text { (iii) } f(x)=\log x \text {. }
$$

4. Theorem: If $f^{\prime}(x)$ be constantly positive, or constantly negative, as $x$ increases from $B$ to $A$, and $a$ be any fixed quantity lying between $\mathrm{A}$ and $\mathrm{B}$, then the expression $\frac{f(x)-f(a)}{x-a}$ constantly increases, or constantly decreases, as $x$ increases from $B$ to $A$ (passing through the value $f^{\prime}(a)$ as $x$ passes through $a$ ). 
Demonstration: Suppose $f^{\prime \prime}(x)$ to be positive. Let $x$ and $y$ be any two quantities lying between the limits $A$ and $B, x$ being greater than $y$. We have to show that

$$
\frac{f(x)-f(a)}{x-a}>\frac{f(y)-f(a)}{y-a} .
$$

First, if $x>y>a$, we have

$$
\frac{f(x)-f(y)}{x-y}>\frac{f^{\prime}(y)-f(a)}{y-a}
$$

Secondly, if $x>a>y$, we have

$$
\frac{f(x)-f(a)}{x-a}>\frac{f(a)-f(y)}{a-y}
$$

Thirdly, if $a>x>y$, we have

$$
\frac{f(a)-f(x)}{a-x}>\frac{f(x)-f(y)}{x-y} .
$$

And in each case the result reduces to

$$
\frac{f(x)-f(a)}{x-a}>\frac{f(y)-f(a)}{y-a} .
$$

If $f^{\prime \prime}(x)$ be negative, the inequality sign is reversed throughout.

Examples : $\quad \frac{x^{m}-a^{m}}{x-a}, \frac{a^{x}-1}{x}, \frac{\tan x}{x}$.

5. Theorem: If $f^{\prime \prime}(x)$ be constantly positive or constantly negative, as $x$ increases from $\mathrm{B}$ to $\mathrm{A}$; and if $a, b, \ldots k$ be any $n$ quantities, not all equal, lying between the limits $A$ and $B$; and $p, q, \ldots t$ be any system of positive multiples corresponding to $a, b, \ldots k$, respectively, then

$$
\frac{p f^{\prime}(a)+q f(b)+\ldots+t f(k)}{p+q+\ldots+t} \geqslant f\left(\frac{p a+q b+\ldots+t k}{p+q+\ldots+t}\right)
$$

according as $f^{\prime \prime}(x) \geqslant 0$, between the limits $A$ and $B$.

Demonstration: Suppose $f^{\prime \prime}(x)$ to be positive. We shall first prove the theorem in the case of two quantities $a$ and $b$. Let $a$ be $>b$, Then $x$ being any quantity between $a$ and $b$, we have

$$
\frac{f(a)-f(x)}{a-x}>\frac{f(x)-f(b)}{x-b} \text {. }
$$


Now, for $x$ write $\frac{p a+q b}{p+q}$. This is permissible as the value of this fraction lies between $a$ and $b$.

Substituting and reducing, we get

$$
\frac{p f(a)+q f(b)}{p+q}>f\left(\frac{p a+q b}{p+q}\right) .
$$

The result is thus proved for two unequal quantities $a$ and $b$. If $a$ and $b$ be equal, the inequality becomes an equality; so that, in any case, we can write

$$
\frac{p f(a)+q f(b)}{p+q} \nless f\left(\frac{p a+q b}{p+q}\right) .
$$

Hence, by induction, we obtain

$$
\frac{p f(a)+q f(b)+\ldots+t f(k)}{p+q+\ldots+t}>f\left(\frac{p a+q b+\ldots+t k}{p+q+\ldots+t}\right) .
$$

If $f^{\prime \prime}(x)$ be negative, the inequality signs are reversed throughout.

Examples :

(i) $f(x)=x^{m}$; (ii) $f(x)=y^{x}$; (iii) $f(x)=\sin ^{x}$; (iv) $f(x)=\tan x$.

[The author then points out that inequalities of a different form can be obtained by writing for $f(x)$, say, $\log f(x)$; so that constancy of sign in $f^{\prime \prime}(x)$ is replaced by that in $u \equiv f(x) . f^{\prime \prime}(x)-\left\{f^{\prime}(x)\right\}^{2}$.

The results are given for this particular case, and a great many interesting results arise out of it.

$$
\begin{aligned}
& \text { E.g., 1. (i) } e^{\frac{1}{x}}<\left(\frac{x}{y}\right)^{\frac{1}{x-y}}<e^{\frac{1}{y}} \text { if } x>y>0 . \\
& \text { (ii) } x^{y-z} \cdot y^{z-x} \cdot z^{x-y}<1 \text { if } x>y>z>0 . \\
& \text { (iii) } x^{\frac{1}{x-1}} \text { constantly decreases as } x \text { increases from } 0 \text { to } \infty \text {, } \\
& \text { passing through the value } e \text { as } x \text { passes through the } \\
& \text { value } 1 . \\
& \text { (iv) }\left(a^{y} . b^{q} \ldots k^{x}\right)^{\frac{1}{p+q+\ldots+t}}<\frac{p a+q b+\ldots+t k}{p+q+\ldots+t} \\
& \text { where } a, b, \ldots k \text { are not all equal, and the symbols all } \\
& \text { denote positive numbers. }
\end{aligned}
$$


2. From $\mathrm{S}_{x} \equiv a^{x}+b^{x}+\ldots+k^{x}$,

(iii) $\left(\frac{a^{x}+b^{x}+\ldots+k^{x}}{n}\right)^{\frac{1}{x}}$ constantly increases as $x$ increases from $-\infty$ to $+x$, and has the limiting value $(a . b, \ldots k)^{\frac{1}{4}}$ when $x=0$.

3. From $\cos x$,

(ii) $(\cos x)^{y-z} \cdot(\cos y)^{i-x} \cdot(\cos z)^{x-y}<1$, if $\frac{\pi}{2}>x>y>z>0$.

(iii) $(\cos x)^{\frac{1}{x}}$ constantly decreases as $x$ increases from 0 to $\frac{\pi}{2}$ and has the limiting value 1 , when $x=0$.

(iv) $(\cos x)^{\nu} \cdot(\cos y)^{y} \cdot<\left(\cos \frac{p x+q y}{p+q}\right)^{p+q}, \frac{\pi}{2}>x>y>0$ and $p$ and $q$ positive.]

On Mathematical Instruments and the accuracy to be obtained with them in some elementary practical problems.

By J. H. A. M'IntYre. 